\title{
A mechanistic meeting point
}

\author{
Scientists from across the chemistry and biology communities often have divergent ideas of what constitutes \\ mechanistic insight into a biological system. Science that forges a middle ground between these disciplines offers \\ the best way forward for chemical biologists.
}

t will come as no surprise to the readers of this journal that the chemical biology community draws from a diverse pool of scientists. Although the next generation of chemical biologists may truly be schooled in two (or even many) disciplines, many 'current' chemical biologists were still formally trained primarily in one subject. As a result, researchers in this multidisciplinary field often have different expectations not only of what scientific questions can and should be asked, but also of how and to what extent they should be answered. Expressed another way, each scientist likely has a different idea of his or her field's standards for mechanistic insight. Because each perspective is based on an informed understanding of the possibilities and limitations inherent to the field, integration of these criteria for interdisciplinary science remains a significant challenge. We suggest that chemists and biologists need to meet at the intersection of these scientific viewpoints to advance research in chemical biology.

Most scientific pursuits can be explained as a search for 'mechanism'. Though the definition of mechanism almost universally corresponds to gaining insight into the what, how and why of the chosen research subject, the specific criteria for establishing a mechanistic hypothesis vary significantly among disciplines. From an organic chemistry perspective, this may correspond to determining a reaction mechanism, which highlights the kinetics and thermodynamics governing the movement of atoms and electrons. From a biochemical perspective, the mechanisms of specific biological processes are mysteries to be solved. Clarifying the identities and functions of the cofactors involved in various enzymatic reactions, for example, is an ongoing challenge. Ask a cell biologist about mechanism, and you'll probably have a discussion about biological pathways that considers the dynamics of biomolecules or organelles in space and time. In a more applied sense, pharmacologists seek to understand the mechanism of action of a potential drug by exploring the interactions of small molecules with biomolecules.

Each of these examples identifies a valid definition of 'mechanism', ranging from which molecules have a role in a specific process to how they behave and finally why they behave as they do. The distinction between these views lies in the level of resolution sought by different disciplines. As a rough approximation, traditional chemical questions can be said to focus on the picometer to nanometer scale, whereas many biochemical questions may extend to the order of micrometers. A biological perspective may lead even further out to millimeters and beyond, as questions about different cell types or species, or even about tissues and organisms, are raised. The cultural blending of the disciplines of chemistry and biology thus requires the integration of these perspectives to fulfill a central aim of chemical biology: to understand biological systems at the molecular level.

Chemical biology questions, then, reflect this desire to push our understanding of cellular pathways and biological processes to greater molecular-level resolution. The challenge then is to determine the best way to gain this insight. In keeping with the multifaceted scope of chemical biology, the 'best' way to answer a question may be to begin with a familiar approach, but continually progress toward meeting the expectations of other scientists in the community. Thus, 'biological' questions should be explored at smaller and smaller scales, whereas the objectives of 'chemical' investigations should be expanded to consider potential and practicality within the natural world. As a journal, we are particularly excited by research that combines diverse philosophical and experimental perspectives to push a field forward.

As a final step, scientists from distinct backgrounds must communicate to each other the excitement of their scientific advances. Because the resulting manuscripts can be diverse both in topic and in experimental approach, at Nature Chemical Biology, we often ask for advice from experts across the scientific spectrum to properly evaluate a given paper. However, as a result, the technical concerns and level of enthusiasm about a manuscript can vary significantly between referees on the same paper. For example, biologists are often concerned with in vivo relevance and eliminating possible alternate explanations given the complexity of the cell. Chemists often express the need for additional characterization of specific molecules or intermolecular interactions in order to provide a sound basis for a particular conclusion. No matter what specific expertise a referee brings to a review, his or her thoughtful and constructive comments provide invaluable guidance in elevating scientific studies to become meaningful interdisciplinary investigations. In any case, integrating or reconciling the reports we receive can be a significant challenge. Our editorial team invests significant effort, through discussion of decisions and establishing editorial criteria for studies across chemical biology, into fairly weighing referee reports and agreeing on reasonable expectations for what constitutes an exciting and influential paper that will be of interest to a general readership of chemists and biologists.

Obviously, it is ideal for scientists from many disciplines to agree that a scientific result is insightful and exciting, because it means that the conclusion crosses scientific borders. Accordingly, chemical biologists should plan their projects and publications to consider each of these fundamentally distinct approaches from the outset. Interaction with colleagues and collaborators with different backgrounds can facilitate these excursions into foreign intellectual territory. In addition, authors should use referee reports as an important guide for developing improved papers that more effectively balance the requirements of research at the interface of chemistry and biology. The integration of expectations concerning mechanistic insight into a new, cohesive, community mind-set will serve as the foundation for chemical biology moving forward. 